Check for updates

Cite this: RSC Adv., 2019, 9, 27369

\title{
Medium optimization based on comparative metabolomic analysis of chicken embryo fibroblast DF-1 cells $\dagger$
}

\author{
Jia Lin, (D) X Xiaoping Yił and Yingping Zhuang*
}

Chicken embryo fibroblast DF-1 cells are increasingly being used in the production of avian virus vaccines. However, the relatively low proliferative capacity does not meet the requirements of industrial production. In this study, we attempted to improve the proliferative capacity of DF-1 cells. The results of intracellular metabolomics showed that 28 types of metabolites could play roles in DF-1 cell growth based on the variance and timing analysis of intracellular metabolites from DF-1 cells grown in two media with distinct growth difference, DMEM/F12 (1:1) and DMEM. By examining the differences in the components in the two media, DOE was used to screen and optimize the growth medium for DF-1 cells. The maximum cell density was $40.72 \%$ higher, and the infectious bursal disease virus (IBDV) titer was 2.68 times higher, in the optimized medium than in the control. This study proposes a complete solution from metabolomics

to media optimization.

Received 9th July 2019

Accepted 15th August 2019

DOI: 10.1039/c9ra05128g

rsc.li/rsc-advances

\section{Introduction}

DF-1 is an immortalized cell line of chicken embryo fibroblasts, arising spontaneously from East Lansing Line (ELL-0) chicken embryos without any endogenous fragments related to avian leukosis virus and sarcoma virus, ${ }^{1}$ which has been demonstrated to have applications in the production of several avian virus vaccines, such as Marek's disease virus, ${ }^{2}$ avian influenza virus $^{3,4}$ and infectious bursal disease virus (IBDV). ${ }^{5}$ DF-1 cells have greater proliferative capacity than the CEF cell line $e^{6}$ but it is still not satisfactory for industrial production; thus, improvement of the reproductive capacity of DF- 1 cells would be valuable.

In addition to the genome, transcriptome and proteome, the metabolome has been widely used in the field of industrial biotechnology in recent years, such as for rational transformation of biological processes, ${ }^{7}$ metabolic network optimization, ${ }^{8}$ in vivo metabolic functional research, ${ }^{9}$ and systemic metabolic engineering. Metabolites can indicate the phenotypes of the actual process, that is, small changes in gene expression or protein activity often lead to substantial changes in metabolites, and these metabolite changes are in turn responsible for cell or tissue behaviour directly while also simultaneously affecting the proteome and transcriptome..$^{10,11}$

State Key Laboratory of Bioreactor Engineering, School of Bioengineering, East China University of Science and Technology, 130 Mei Long Road, Xu Hui District, Shanghai 200237, China. E-mail: ypzhuang@ecust.edu.cn; Tel: +862164253337

$\dagger$ Electronic supplementary information (ESI) available. See DOI: 10.1039/c9ra05128g

\$ These authors contributed equally.
Therefore, the metabolome can reflect the physiological characteristics of the cell and identify the bottleneck in cell cultivation and metabolism to improve the efficiency of biological processes. In terms of analysis, metabolite measurements only require classic analytical chemistry techniques and are the least expensive among all omics methods. In addition, analytical methods are suitable for different organisms and species and the results are acceptable for comparison of different conditions and cell types directly. ${ }^{12}$ In short, the metabolome can provide a large amount of detailed information regarding biological process phenotypes and biosystems, which cannot obtain by other omics methods. Korneli C. et al. identified a dynamic trend in intracellular amino acids in Bacillus megaterium in scale-down simulation experiments by metabolomics and identified four types of amino acids as biomarkers, which provided important clues regarding the feeding strategy. ${ }^{13}$ Chrysanthopoulos et al. applied metabolomics to accurately monitor the BHK culture process. ${ }^{14}$ With all other conditions remaining constant, different bioreactors, culture sizes and the cell generations showed the different physiological characteristics in terms of the metabolome. Biomarkers from different culture processes can be identified by metabolomics to optimize and accurately monitor the cell culture process.

Design of Experiment (DOE) is a common method for factor optimization and to decrease the number of experimental steps via decreased factor counts and levels in biological process optimization. There are many variable factors in actual biological processes, but not all factors have significant impacts. Therefore, the first step in biological process optimization is identification of significant factors. Plackett-Burman design (PBD) is a two-level fractional factorial design developed by 
Plackett and Burman and has been extensively used for screening important factors from pre-analysed results and estimating the extent of the effect with relatively few trials; however, this design is not quantitatively accurate. ${ }^{15}$ The BoxBehnken design (BBD) is an experimental design for the secondorder polynomial response surface method that is widely used to estimate the response region; $\mathrm{BBD}$ is a three-level fractional factorial design developed by Box and Behnken and provides mathematical models with the dependence degree of each independent variable (each component concentration in medium or the operating parameters), including the predicted result of each variable level. ${ }^{16}$ In summary, DOE is a useful tool for medium optimization because of the reduced research cost, ${ }^{17,18}$ reduced optimization time, ${ }^{19}$ improved product quality and increased target product yield ${ }^{20-23}$ associated with this method.

This study first applied metabolomics to screen nutrients that influenced the growth of DF- 1 cells and then determined significant key factors by $\mathrm{PBD}$, selected the optimal range by one-factor-at-a-time design, and finally estimated the response level and the relationship between factors so that the optimal conditions for cell growth and the optimal effects could be determined according to the second-order polynomial model by BBD. Based on metabolomics and the DOE methodology, we established a strategy for optimization of cell culture medium.

\section{Methods}

\section{DF-1 cell culture}

Routine cell culture of adherent DF-1 cells was performed in a 25 $\mathrm{cm}^{2}$ Nunc EasYFlask (Thermo Scientific) with $5 \mathrm{~mL}$ of DMEM/F12 (1:1) or DMEM (Gibco) with 5\% fetal bovine serum (Biological Industries) in a humidified incubator at $37{ }^{\circ} \mathrm{C}$ with $5 \% \mathrm{CO}_{2}$. Cell number and viability were determined using a Countstar (ALIT Life Science), an automated trypan blue cell counter.

\section{IBDV strain and preparation}

IBDV was used to infect DF-1 in flasks when the cells reached $90 \%$ confluence, and the cells were harvested when $80 \%$ of the cells exhibited lesions; virulence was determined using TCID $_{50}$.

\section{Experimental design and procedure of metabolome}

For metabolomic analysis of global biochemical profiles, cells were cultivated in a $75 \mathrm{~cm}^{2}$ Nunc EasYFlask with $15 \mathrm{~mL}$ medium, and a total of $10^{7}$ cells were harvested for each medium and stored at $-80{ }^{\circ} \mathrm{C}$ immediately after washing with ice-cold phosphate-buffered saline. Three of these cultures were used to determine the intracellular metabolites at each time point ( $t=12,24,36$, and $72 \mathrm{~h}$ ). Metabolite determination was performed by Metabolon, Inc. (Durham, NC) using standard protocols. Metabolon has developed a platform that integrates the chemical analysis, metabolite identification and relative quantification, data reduction, and quality assurance components of the process. The methodology has been detailed elsewhere. ${ }^{24}$ Individual cell samples ( $n=3$ per group) were extracted and split into equal parts for analysis on the GC/MS and UPLCMS/MS platforms.

Prior to statistical analysis, the data obtained were normalized to the protein concentration by Bradford analysis. ${ }^{25}$ All identified metabolite relative abundance matrices were uploaded to MetaboAnalysis (http:/www.metaboanalyst.ca) for multivariate statistical data analysis and the pathway analysis. ${ }^{26,27}$

\section{Statistical analysis strategy}

Plackett-Burman design. In this study, PBD was used to screen the factors that significantly affected cell growth from twenty-eight metabolites to improve the maximum cell density obtained and the specific growth rate in logarithmic growth phase, including twenty-one metabolites based on metabolomics and seven metabolite components from differences in the components in the comparative medium, and classified into 10 categories as listed in Table 1 according to possible pathways. Each variable was tested at two levels-high $(+1)$ and low $(-1)$ which were initially experimentally estimated in DMEM/ F12 (1: 1). In summary, eleven independent variables (ten of the abovementioned variables and one dummy variable) were screened by twelve trial runs according to PBD with $N=11$. Further details regarding the PBD matrix are listed in Table S4. $\dagger$

One-factor-at-a-time design. A one-factor-at-a-time design was used to determine the optimal range of values. The three most significant factors obtained from the PBD experiment were further optimized by one-factor-at-a-time experiments for response surface analysis.

Box-Behnken design. Response surface analysis applied $\mathrm{BBD}$ for evaluation of the effects of three independent variables

Table 1 Metabolites classification and component concentration in Plackett-Burman design

\begin{tabular}{|c|c|c|c|c|}
\hline \multirow[b]{2}{*}{ Symbol } & \multirow[b]{2}{*}{ Group } & \multirow[b]{2}{*}{ Components } & \multicolumn{2}{|l|}{ Level (mM) } \\
\hline & & & -1 & 1 \\
\hline \multirow[t]{2}{*}{ A } & \multirow[t]{2}{*}{ Amino acids 1} & Arginine & 0.7 & 1.4 \\
\hline & & Proline & 0.15 & 0.3 \\
\hline \multirow[t]{3}{*}{ B } & \multirow[t]{3}{*}{ Amino acids 2} & Glycine & 0.25 & 0.5 \\
\hline & & Serine & 0.25 & 0.5 \\
\hline & & Threonine & 0.45 & 0.9 \\
\hline \multirow[t]{2}{*}{$\mathrm{C}$} & \multirow[t]{2}{*}{ Amino acids 3} & Aspartate & 0.05 & 0.1 \\
\hline & & Asparagine & 0.05 & 0.1 \\
\hline \multirow[t]{2}{*}{$\mathrm{D}$} & \multirow[t]{2}{*}{ Amino acids 4} & Glutamate & 0.05 & 0.1 \\
\hline & & Glutamine & 2.5 & 5 \\
\hline \multirow[t]{3}{*}{$\mathrm{E}$} & \multirow[t]{3}{*}{ Amino acids 5} & Histidine & 0.15 & 0.3 \\
\hline & & Cysteine & 0.1 & 0.2 \\
\hline & & Methionine & 0.12 & 0.23 \\
\hline $\mathrm{F}$ & Putrescine & Putrescine & $5.03 \times 10^{-4}$ & $1.01 \times 10^{-3}$ \\
\hline $\mathrm{G}$ & Nucleotide & Nucleotides & $1.51 \times 10^{-3}$ & $3.02 \times 10^{-3}$ \\
\hline \multirow[t]{2}{*}{$\mathrm{H}$} & \multirow[t]{2}{*}{ Vitamins } & Biotin & $1.43 \times 10^{-5}$ & $2.87 \times 10^{-5}$ \\
\hline & & VB12 & $5.02 \times 10^{-4}$ & $1.00 \times 10^{-3}$ \\
\hline \multirow[t]{3}{*}{$\mathrm{J}$} & \multirow[t]{3}{*}{ Inorganic salts } & $\mathrm{Zn}^{2+}$ & $1.50 \times 10^{-3}$ & $3.00 \times 10^{-3}$ \\
\hline & & $\mathrm{Cu}^{2+}$ & $5.20 \times 10^{-6}$ & $1.04 \times 10^{-5}$ \\
\hline & & $\mathrm{Fe}^{2+}$ & $1.50 \times 10^{-3}$ & $3.00 \times 10^{-3}$ \\
\hline \multirow[t]{2}{*}{$\mathrm{K}$} & \multirow[t]{2}{*}{ Others } & Linoleic acid & $1.50 \times 10^{-4}$ & $3.00 \times 10^{-4}$ \\
\hline & & Lipoic acid & $5.10 \times 10^{-4}$ & $1.02 \times 10^{-3}$ \\
\hline $\mathrm{L}$ & Dummy & & 0 & 0 \\
\hline
\end{tabular}


on cell growth to achieve increased maximum cell density and specific growth rate in the logarithmic growth phase. With the other factors maintained in DMEM/F12 (1:1), we studied the three significant variables at the three coded levels: high $(+1)$, intermediate (0) and low levels $(-1)$. To determine the optimum values for the three selected variables, 15 trial runs were designed by BBD, including 3 replicates. The experimental matrix, including the experimental and predicted results, is provided in Table $\mathrm{S} 6 . \dagger$

The following second-order polynomial model fits the relationship between the response and the test variable. The equations obtained using statistical methods are as follows:

$$
Y=\beta_{0}+\sum_{i=1}^{3} \beta_{i} X_{i}+\sum_{i=1}^{3} \beta_{i i} X_{i}^{2}+\sum_{i=1}^{2} \sum_{j=i+1}^{3} \beta_{i j} X_{i} X_{j}
$$

where $Y$ is the predicted response and $\beta_{0}, \beta_{i}, \beta_{i i}$, and $\beta_{i j}$ are the constant, linear coefficient, quadratic coefficient and interaction coefficient, respectively. $X_{i}$ and $X_{j}$ are the independent variables.

Validation of the fitting model. The second-order polynomial described above was used to direct the experiment to validate the fitted model. The DF-1 cells cultured in optimized medium validate the optimization results using the statistical strategy for the maximum cell density obtained and the specific growth rate in the logarithmic growth phase as the targets compared with cells cultured in DMEM/F12 (1:1) medium as the control.

Method matrix and ANOVA. The experimental method matrix was designed throughout the process, including in PBD and BBD, and the restricted analysis of all the experimental data was performed using Design Expert 10.0.4 (Stat-Ease Inc., Minneapolis, USA). The statistical significance of the variables was evaluated by applying analysis of variance (ANOVA) using Student's $t$-test. The adequacy of the model was verified using Fisher's $F$-test.

\section{Results}

\section{Cell growth}

The specific growth rate and maximum cell density are two important indicators of the cell growth. DF-1 cells were cultured in DMEM/F12 $(1: 1)$, with a maximum specific growth rate of $0.033 \mathrm{~h}^{-1}$ (Fig. 1a) and a maximum cell density of at least $1.63 \times$ $10^{6}$ cells per ml, and in DMEM, with a maximum specific growth rate of $0.029 \mathrm{~h}^{-1}$ and a maximum cell density of only $0.67 \times 10^{6}$ cells per ml. There was a significant difference in the growth of DF-1 cells inoculated at the same cell density in DMEM/F12 (1:1) and DMEM. DF-1 cells had a high specific growth rate and could be maintained for long duration in the logarithmic growth phase DMEM/F12 (1:1) medium; thus, a high maximum cell density was achieved in this medium.

\section{Metabolome analysis}

Multivariate statistical analysis at different time points. According to the growth of DF-1 cells in DMEM/F12 (1:1) and DMEM (Fig. 1a), ten million cells, in triplicate, were collected for metabolite measurement at $12 \mathrm{~h}, 24 \mathrm{~h}, 36 \mathrm{~h}$ or $72 \mathrm{~h}$, and the differences in cell growth between the two media increased with time. The complete metabolite analysis process included analysis of the lag phase and logarithmic growth phase of cell growth. We obtained 193 intracellular metabolites, including amino acids, peptides, carbohydrates, lipids, nucleotides, cofactors, and vitamins. LC/MS and GC/MS were used for intracellular metabolite determination to ensure that reliable data were selected for analysis (Table S1†).

Hierarchical clustering was performed to classify the detected metabolites as a heat map (Fig. 1b). Principal component analysis (PCA) (Fig. 1c) showed that the metabolite samples could be separated based on different media types and sampling time points, indicating that the intracellular pools reflected the extracellular environment and that the data regarding the structure and quality of the detected metabolites were suitable for statistical inference and monitoring of the cell culture process.

To study the metabolites associated with DF- 1 cell growth in the two media, the variation in the intracellular metabolites of DF-1 cells was analysed. Because the differences in intracellular metabolites at the $36 \mathrm{~h}$ and $72 \mathrm{~h}$ sampling points were the most significant among the four sampling points when DF-1 cells were grown in DMEM/F12 (1:1) and DMEM (Fig. 1a), respectively, pathway analysis for the two sampling points was performed. The results showed that the nine pathways were significantly different at both sampling points, the pathway effects of which were higher than 0.18 , and the $p$-values were lower than 0.05 (Fig. 2). Therefore, supplementation of nucleotides, cysteine, methionine, lysine, glycine, serine, and threonine could promote the growth of DF- 1 cells.

Metabolite timing analysis of DF-1 cells in two media. To identify the metabolites linked with DF-1 cell growth throughout the culture, we performed interactive principal component analysis (iPCA) of the intracellular metabolites in both media in four sampling times. Two heat maps were generated for 50 selected metabolites that changed significantly over time, and the maps showed that the intracellular metabolite levels clearly changed over time and that the results were suitable for analysis (Fig. 3).

The pathway analysis further integrated the KEGG pathway database, and the metabolome visualization analysis showed the metabolic pathways which changed significantly in the DF-1 cells over time in both media. The $p$-values from the pathway abundance analysis showed all the discrepant pathways and pathway topology analysis identified the pathway effects. Common metabolic pathways with significant changes in DF-1 cells over time in both media were identified $(p<0.01)$, and pathways effects greater than 0.18 were selected for further analysis (Table S2 $\dagger$ ). The common metabolic pathways screened included the twenty-two metabolites identified by the above analysis.

The $p$-values for pathway abundance analysis and the impact values calculated by pathway topology analysis are listed in Table S2. $\uparrow$ We found that the $p$ value of the aminoacyl-tRNA synthesis pathway was 0.002 in DMEM/F12 (1:1), while the $p$ value among the pathways was 0.005 in DMEM, indicating that multiple amino acids affected DF-1 cell growth in both media and that amino acid metabolism could be one of the main factors affecting cell growth. The most significant amino acids included arginine, proline, glycine, serine, threonine, alanine, aspartate, glutamate, histidine, cysteine and methionine. 
a

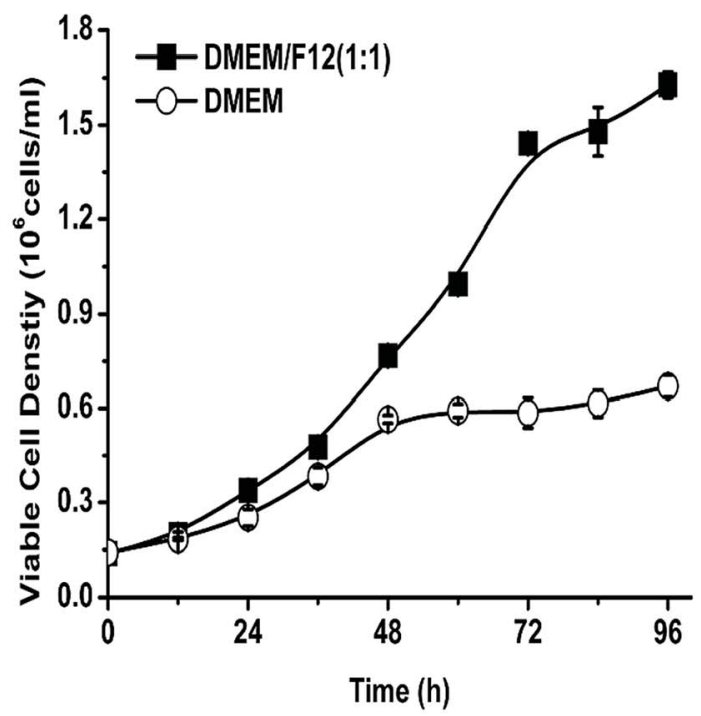

b

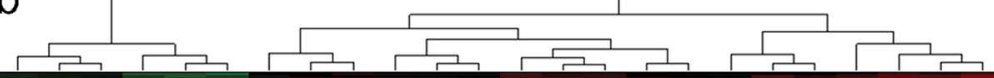

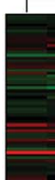
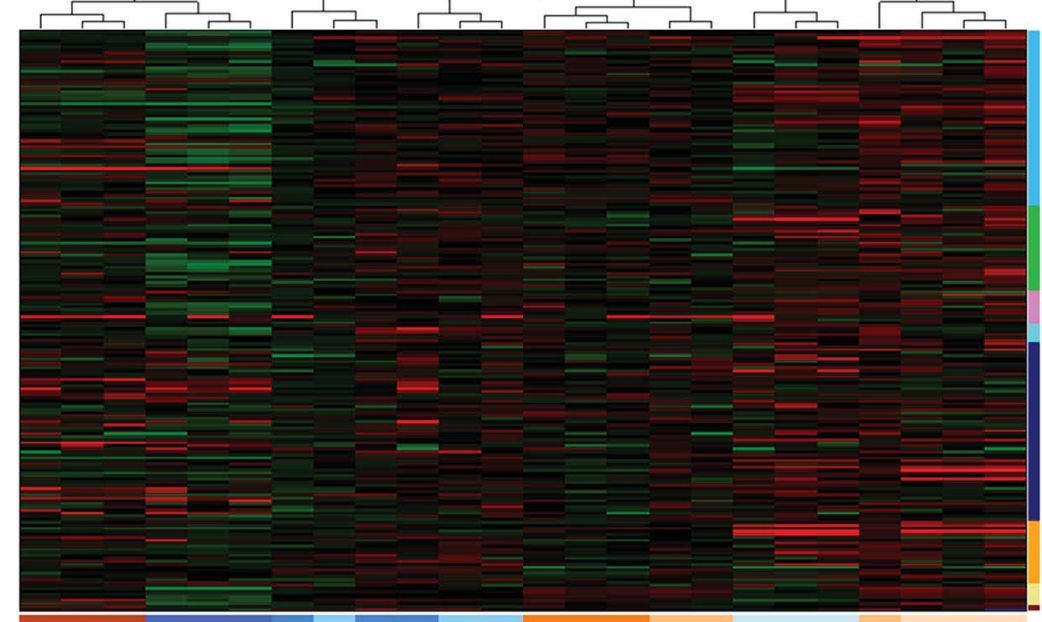

C

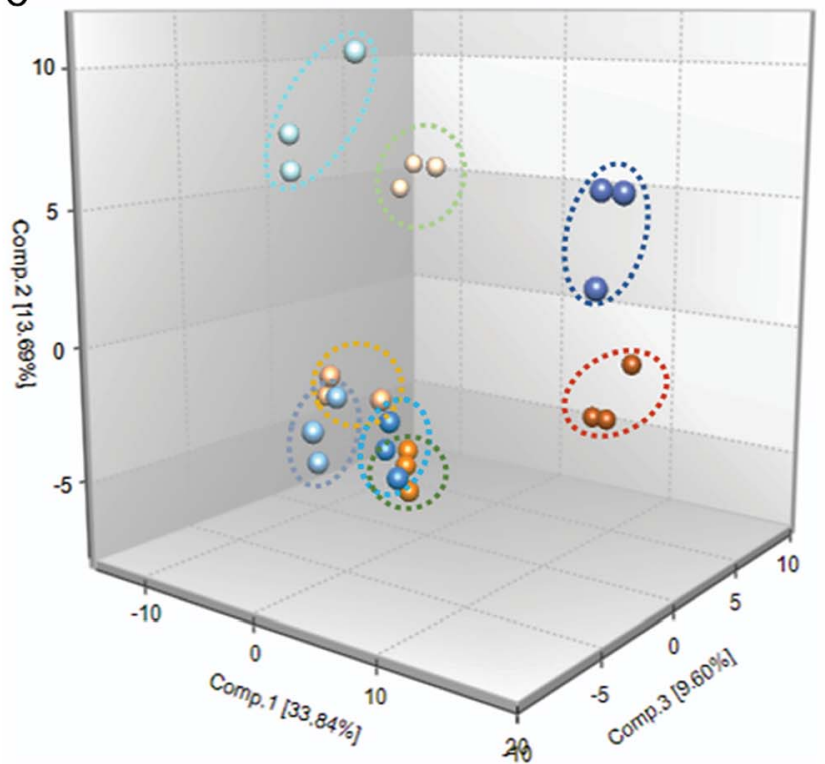

Complete Euclidean Cluster 3.00
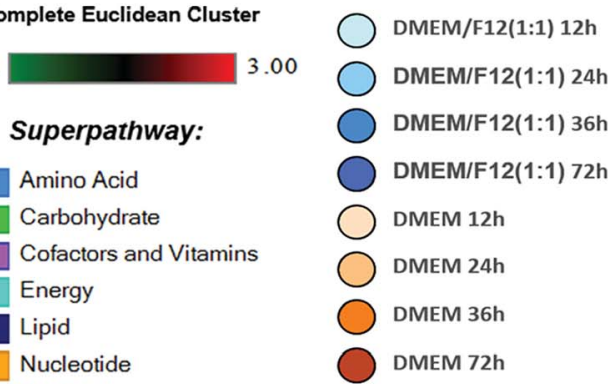

Superpathway:
Amino Acid
Carbohydrate
Cofactors and Vitamins
Energy
Lipid
Nucleotide
Peptide
Xenobiotics

Fig. 1 DF-1 cells in DMEM/F12 (1:1) and DMEM. (a) Viable cell density of DF-1 cells in DMEM/F12 (1:1) and DMEM (0-96 h); (b) threedimensional principle component analysis (PCA) plot of 193 metabolite features. Comp. 1 accounted for 33.84\% of the variation between the eight groups and comp. 2 for $13.69 \%$; (c) hierarchical clustering displaying the features expression pattern. Each column represents one biological sample and each horizontal line represents one metabolite feature. DF-1 cells were maintained either in DMEM/F12 (1:1) or DMEM for $12 \mathrm{~h}, 24 \mathrm{~h}, 36 \mathrm{~h}$, or $72 \mathrm{~h}$ then harvested for metabolomic profiling and three biological replicates of each group were provided. Application of PCA and hierarchical clustering provided by Metabolon to determine separation of individual samples as a function of cellular metabolites demonstrated greater segregation between growth DMEM/F12 (1: 1) and DMEM at different time points indicating that both growth medium and time in culture had a profound impact on global metabolism.

In addition, purine metabolism and pyrimidine metabolism had a similar effect on growth in DMEM/F12 (1:1) and DMEM, with effects of 0.42 and 0.30 observed by topological analysis (Table S3†), respectively. Nucleotides are important for cellular metabolism, acting as building blocks for the synthesis of RNA or DNA and providing energy in many reactions. In both media, the intracellular relative levels of mononuclear acids were substantially reduced during cell growth and significantly decreased at $72 \mathrm{~h}$, while nucleotides reached relatively high levels at $72 \mathrm{~h}$, indicating that continuous synthesis of nucleotides is required for maintenance of cell expansion during the growth process; we speculated that provision of nucleotides or nucleotide synthesis precursors could promote DF-1 cell growth.

Determination of the components to be optimized. The components to be optimized were determined by integrating the intracellular metabolome of DF-1 cells grown in DMEM/F12 (1:1) and DMEM with the comparation between the components of the two media, and categorized into 10 groups according to component properties and pathways with similar function in the promotion of cell growth in the KEGG pathway database (Table 1).

Optimization of cell growth medium based on DOE

Screening of significant factors for cell growth by Plackett-Burman design. The PBD was applied to the 10 groups as mentioned 
a

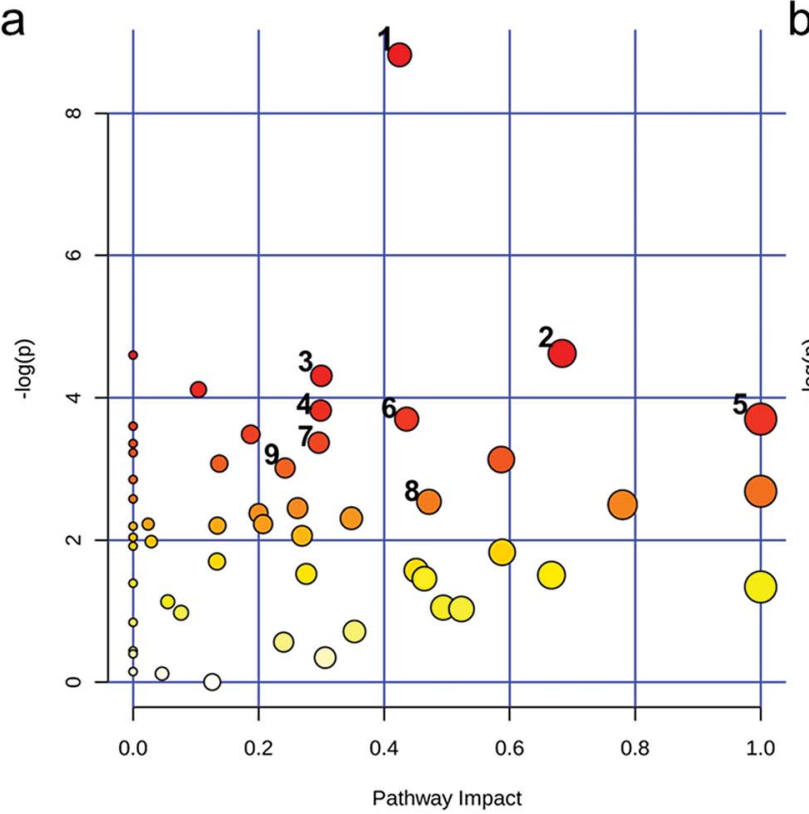

b

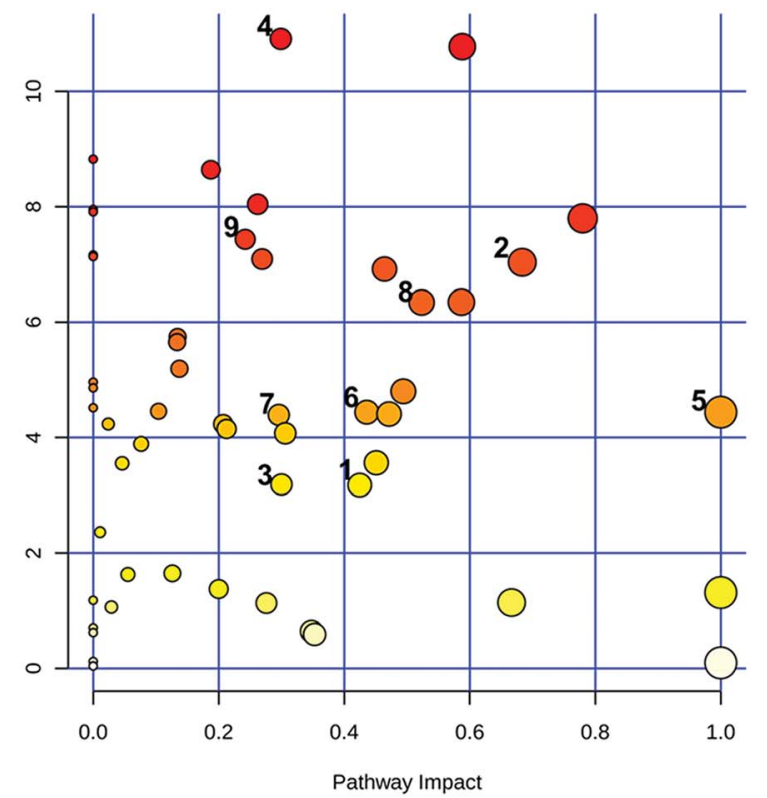

Fig. 2 Differential metabolic pathways of DF-1 cells in the two media: (a) DMEM/F12 (1: 1); (b) DMEM. The metabolome shows all matched pathways according to the $p$ values from the pathway enrichment analysis ( $y$-axis and circle colour) and pathway impact values from the pathway topology analysis ( $x$-axis and circle size). The numbers besides the circles represented the differential metabolic pathways: 1: purine metabolism; 2: cysteine and methionine metabolism; 3: lysine biosynthesis; 4: pyrimidine metabolism; 5: phenylalanine, tyrosine and tryptophan biosynthesis; 6: phenylalanine metabolism; 7: sphingolipid metabolism; 8: glycine, serine and threonine metabolism; 9: glycerophospholipid metabolism.

above with the targets of maximum cell density and maximum specific growth rate, as shown in Fig. 4a and Table S4. $\dagger$ The restriction model with maximum cell density as the target showed a $p$-value less than 0.0001 , an $F$ value of 47.42 , a multivariate correlation coefficient $R^{2}$ of 0.9646 , and a model variable range of $13.89 \%$, indicating that the model fits the entire restriction range well. The restriction model with the maximum specific growth rate as the target had a $p$-value less than 0.0001, an $F$ value of 53.89, a multivariate correlation coefficient $R^{2}$ of 0.9685 , and a variable range of $15.51 \%$, indicating that the model could also fit the entire restriction range well. A positive or negative coefficient in one factor in the restriction model indicated a positive or negative influence of this factor on the response. Among the 10 groups of components tested, 4 groups of components with significant influence were determined with the target of maximum cell density (Fig. 4b), and 4 groups of components with significant effects were also identified with the target of maximum specific growth rate (Fig. 4c). In the model with maximum cell density as the target, the amino acid 5 group and vitamins group had significant positive effects, while the amino acid 1 group and inorganic salt group had significant negative effects. In the model with the maximum specific growth rate as the target, the amino acid 5 group and the other group had significant positive effects, while the inorganic salt group and amino acid 4 group had significant negative effects. Considering that the concentrations of the negative components in the DMEM/F12 (1:1) and DMEM did not inhibit cell growth significantly, the amino acid 5 , vitamin and other groups were further analysed and optimized in the subsequent study.
One-factor-at-a-time design. According to the PBD experimental results, the acid 5 , vitamin and other groups, which significantly affected cell growth, were set to three concentration levels from low to high, and the concentrations of the other factors were the same as the original concentration in the DMEM/F12 (1:1), as shown in Fig. $4 \mathrm{~d}$ and Table S5. $\dagger$ The experiment with the amino acid 5 yielded a high maximum cell density at level 1.5 , while the difference in the maximum specific growth rate at the three levels was not significant, so the range of level 1 to level 1.5 was selected for subsequent studies. The experiment with the vitamin group also achieved a high maximum cell density at level 1.5, and simultaneously, a high maximum specific growth rate was obtained, so the range of level 1 to level 1.5 was selected for subsequent studies. The experiment with the other group had a high maximum cell density at level 1.5 , while the maximum specific growth rate was the highest at level 2. Considering the effect of the growth rate on virus reproduction, level 1 to level 2 was selected for subsequent studies with the other group.

Box-Behnken design. The BBD results (Fig. 4e and Table S6†) were evaluated to identify the interactions between significant factors and determined the optimal levels of the three significant factors based on the results of the PBD experiments and the one-factor-at-a-time experiments. The variance analysis of the restriction model showed that the quadratic equation models based on the maximum cell density and the maximum specific growth rate were significant (model I based on maximum cell density, $F=5.24, p<0.05$; model II based on maximum specific growth rate, $F=3.95, p<0.05$ ). For model I, the analytical coefficient $R^{2}$ was 0.9041 , indicating that the measurement results and the predicted results were consistent, 

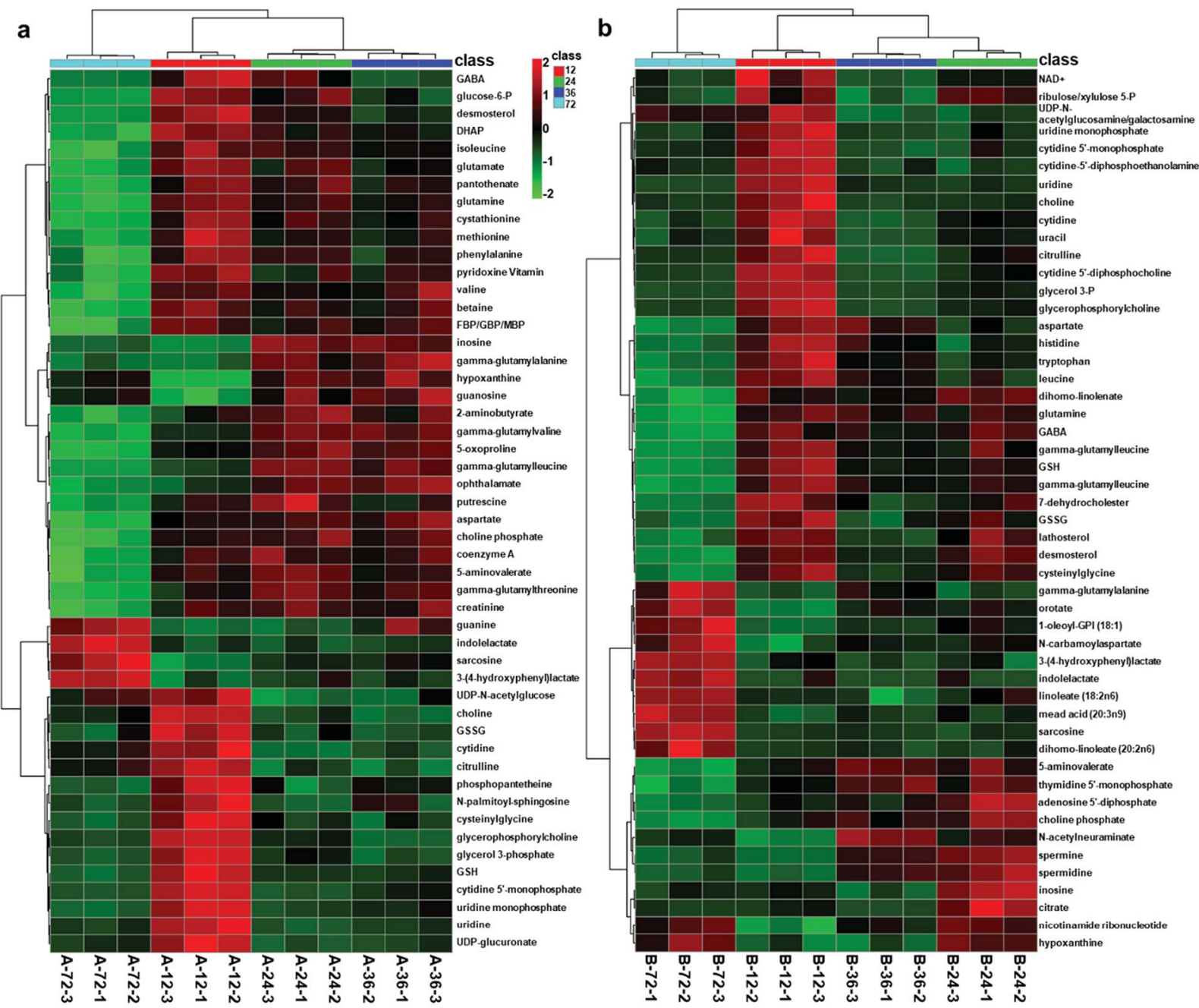

Fig. 3 Heatmaps illustrating intracellular metabolite timing analysis of DF-1 cells in the two media: (a) DMEM/F12 (1 : 1); (b) DMEM. The top 50 features were ranked using the $t$ test, distance was measured using the Pearson correlation, and clustering was determined using the Ward algorithm.

and the Adj $R^{2}$ values showed that the model could explain a variation of $73.14 \%$ (Fig. $4 \mathrm{f}-\mathrm{h}$ ). However, for model II, the analytical coefficient $R^{2}$ was 0.7479 , and the $\operatorname{Adj} R^{2}$ values showed that the model could only explain a difference of $55.86 \%$ (Fig. 4i-k), indicating insufficient correlation between measured and predicted values. Based on the significant differences between model I and model II, the individual effects of the amino acid 5, vitamin, and other groups on maximum cell density and maximum specific growth rate were not all significant, whereas the curved surface effects were significant. The interaction effect and the optimization level of the variables were determined according to the response surface points.

For model I, the correlation coefficient was obtained from restriction analysis of the multivariate quadratic equation as follows:

Maximum cell density

$$
\begin{aligned}
& =2.66 \times 10^{6}+1.79 \times 10^{5} \times A+5.88 \times 10^{4} \times B+2.5 \\
& \times 10^{3} \times C-1.3 \times 10^{5} \times A B+2.25 \times 10^{4} \times A C \\
& +7.5 \times 10^{3} \times B C-9.42 \times 10^{4} \times A^{2}-1.69 \times 10^{5} \\
& \times B^{2}-1.27 \times 10^{5} \times C^{2}
\end{aligned}
$$

and for model II, the correlation coefficient was obtained from interaction restriction analysis of the model equation as follows:

Maximum specific growth rate

$$
\begin{aligned}
& =0.035+0.00053 \times A+0.0010 \times B-0.0058 \\
& \times C-0.0015 \times A B+0.0012 \times A C-0.0025 \times B C,
\end{aligned}
$$

where $A, B$, and $C$ represent the coded levels of the amino acid 5 group, vitamin group and other group, respectively, and their actual values are presented in Table 1.

Experimental verification. The three-dimensional analysis of the response surface indicated that the model had a maximum cell density and a corresponding specific growth rate, as shown in Fig. $4 \mathrm{f}-\mathrm{k}$. The maximum cell density predicted was $2.68 \times 10^{6}$ cells per $\mathrm{ml}$, and the maximum specific growth rate was $0.038 \mathrm{~h}^{-1}$, with the levels of each component group determined to be as follows: amino acid 5 group $=1$, vitamin group $=-0.78$, other group $=0.86$. Compared with the values in the original medium, the maximum cell density and maximum specific growth rate were significantly improved. 


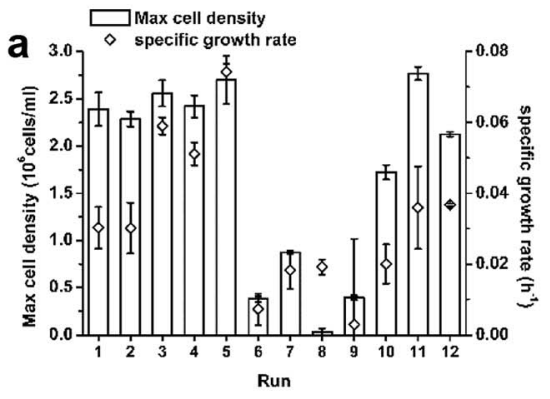

d

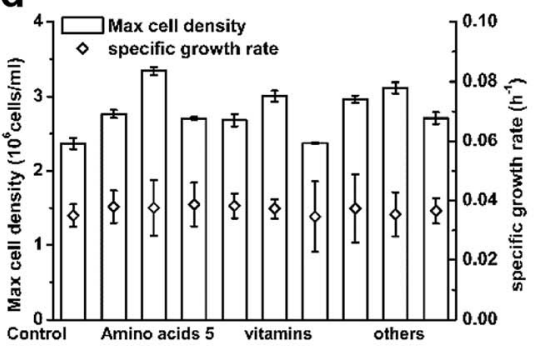

f

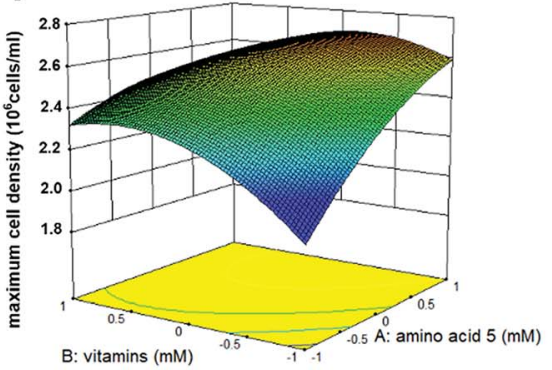

i

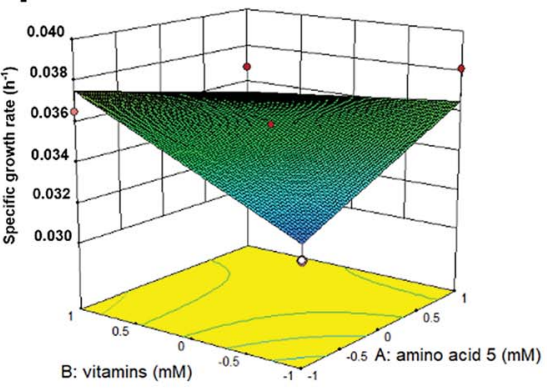

j
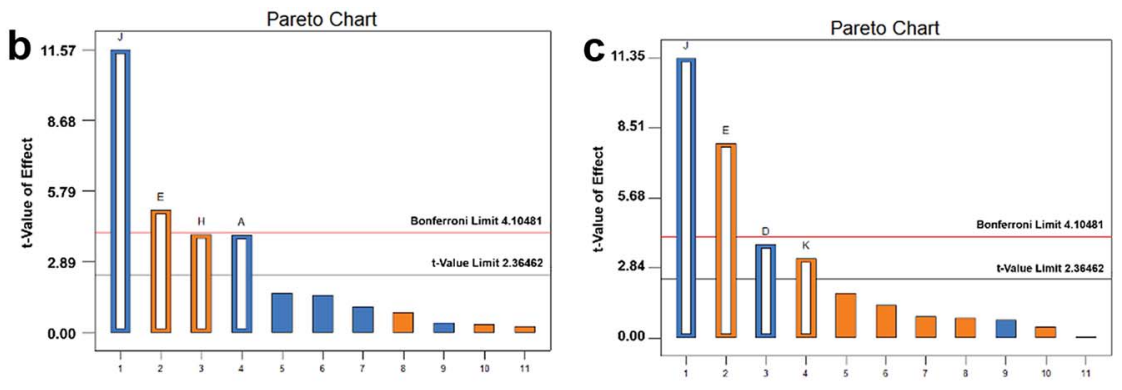

raon

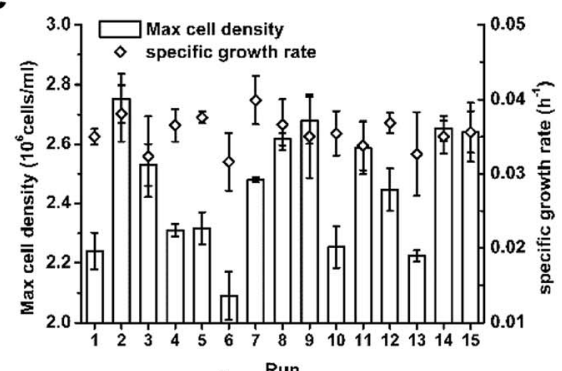

h
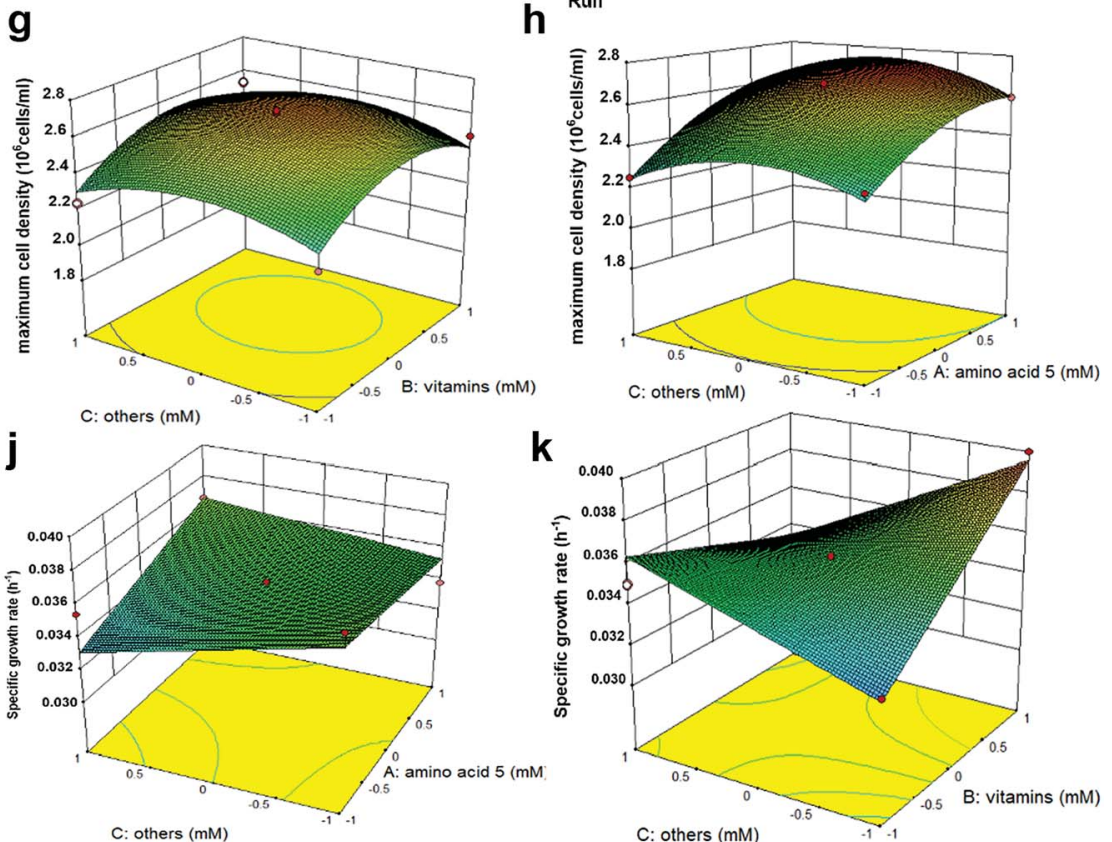

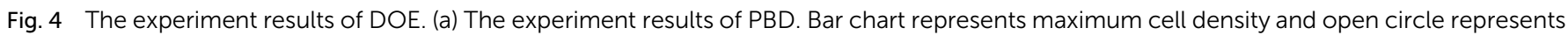

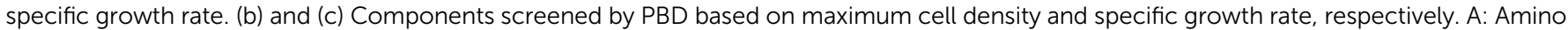

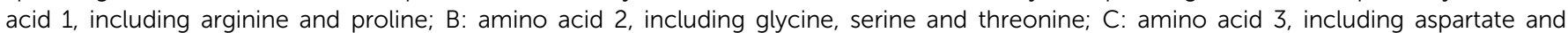

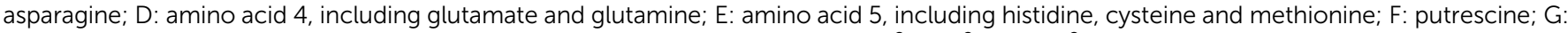

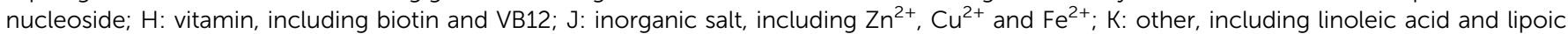

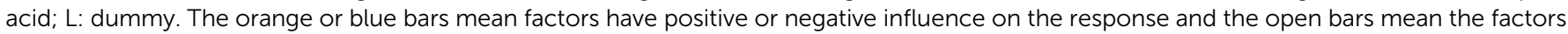

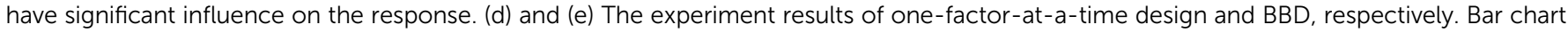

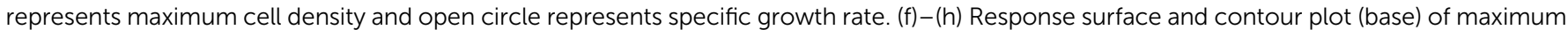

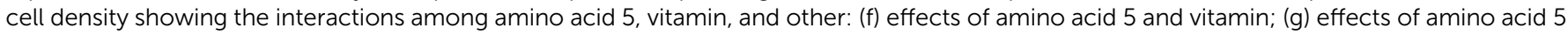

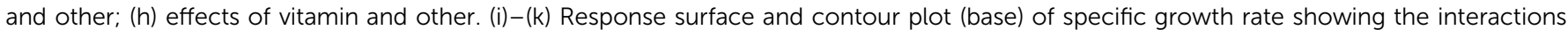

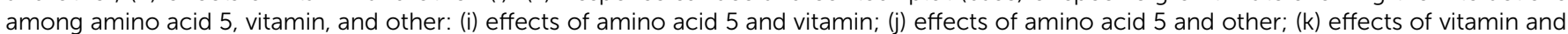
other. $N=3$ biological replicates, and error bars represent s.d.

When DF-1 cells were cultured in the optimized growth medium, the maximum cell density increased by $40.72 \%$ compared with the control (DMEM/F12 (1:1)) (Fig. 5a), and the maximum specific growth rate increased by $13.7 \%$, which was similar to the predicted values. Simultaneously, the IBDV titer obtained for DF- 1 cells cultured in the optimized medium significantly increased to 2.68 times higher than that of the control (Fig. 5b). 
a

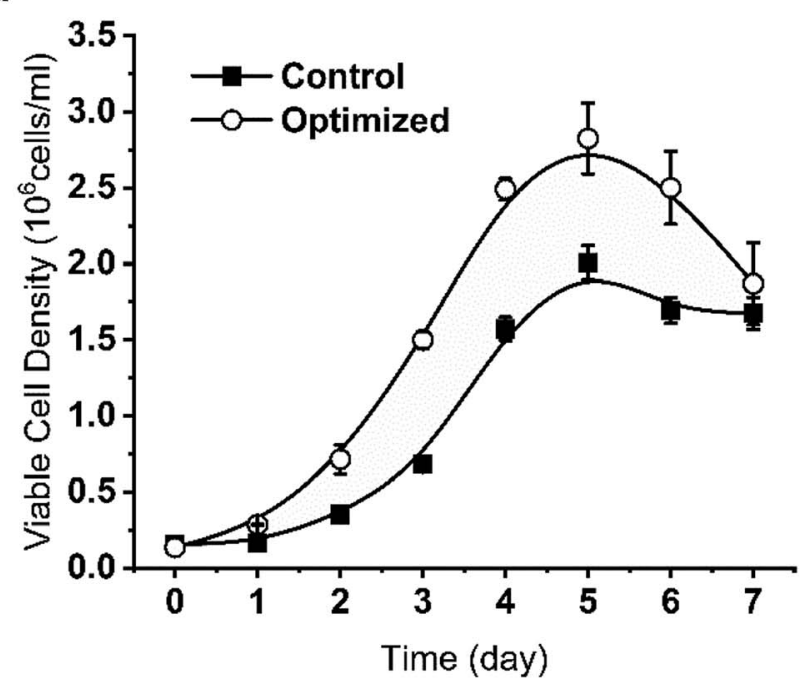

b

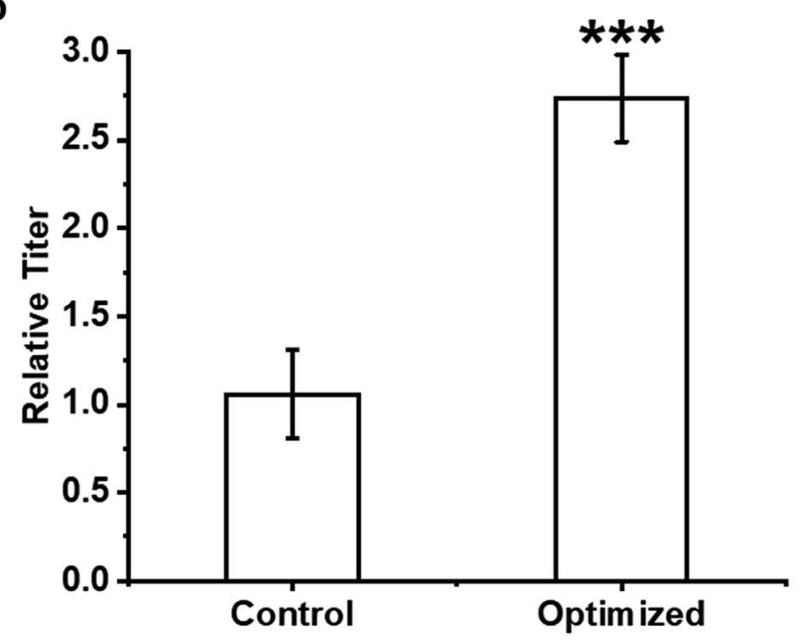

Fig. 5 Comparison of production between the optimized and control media. The asterisks represent $p$ value calculated by two-ANOVA analysis $-* * * p=0.002 . N=3$ biological replicates, and error bars represent s.d.

\section{Discussion and conclusions}

Metabolites are sensitive to genomic changes and cellular responses to external stimuli. Therefore, the metabolomics has potential advantages in the optimization of cell growth. In recent years, due to the considerable advances in routine analytical techniques and animal cell metabolomics, a specific map depicting the cellular metabolic state can be generated for optimization of the cell culture process optimization by measurement of specific changes in intracellular metabolites.

There were significant differences in the growth of DF-1 cells in DMEM/F12 (1:1) and DMEM. To investigate the specific changes in cell metabolism in two culture conditions and the effects of different nutrient components on cell growth, intracellular metabolomes were analysed between the two media. The PCA results showed that the bioparallel sample groups were well aggregated and could be differentiated based on the type of medium and sampling time, indicating that the data could be used to monitor the DF-1 cell culture process.

Combined with the time series analysis, differential analysis of the metabolites and pathways revealed that amino acid metabolism plays an important role in DF-1 cell growth. Amino acids are important for cell metabolism, not only in protein metabolism, but also as precursors of various macromolecular metabolites. For example, cysteine is involved in the synthesis of glutathione and regulates the cellular redox state; ${ }^{28,29}$ methionine is one of the sources of methyl groups in biomethylation reactions and plays an important role in the methylation of DNA and histones. ${ }^{30}$ In proliferating cells, amino acids contribute more than glucose to biomass synthesis. Hosios et al. used [U- ${ }^{14} \mathrm{C}$ ]-labeled amino acids to show labelling of most of the carbon skeleton in proliferating animal cells, verifying that amino acids primarily participated in protein and nucleotide synthesis, while glucose exhibited increased participation in biomass synthesis only in the absence of amino acids. ${ }^{31}$ The metabolomic analysis in this study showed that glycine, serine, threonine, aspartate, asparagine, glutamate, and glutamine might be involved in central carbon metabolism via different metabolic pathways, and in turn in biomass synthesis, indicating that amino acids might affect the synthesis of cellular biomass by regulating protein anabolism or by being directly involved in protein synthesis.

The results of the DOE methodology showed that biotin, cobalamin (VB12), linoleic acid and lipoic acid had significant effects on the growth of DF-1 cells. Biotin is involved in the metabolism of glucose, amino acids and fatty acids, covalently binding to coenzyme synthase (HCS) as the coenzyme of four carboxylases (pyruvate, propionyl-CoA, 3-methyl crotonyl-CoA, and acetyl-CoA) in animal cell culture in vitro. ${ }^{32} \mathrm{VB} 12$ is a complex organometallic cofactor that is associated with several enzymes that are involved in many types of cellular metabolism. ${ }^{33,34}$ Linoleic acid, the crucial ligand for nuclear receptors in animal cell lipogenesis and fat deposition, affects the expression of genes associated with animal cell proliferation and lipid catabolism with eicosatetraenoic acid. Liu et al. cultured duck primary hepatocytes with linoleic acid and eicosatetraenoic acid, which resulted in promotion of cell growth. ${ }^{35}$ Lipoic acid enhances the cellular antioxidant capacity, scavenges free radicals, promotes fat consumption, and inhibits oxidative stress. ${ }^{36}$ The results of the response surface design indicated that promotion of the utilization of lipid metabolites was beneficial for DF-1 cell growth.

In this study, the intracellular metabolites of DF-1 cells grown in DMEM/F12 (1:1) and DMEM were first analysed by difference analysis and time series analysis. It was inferred that amino acid metabolism and lipid metabolism played important roles in cell growth. By examining the differences in the components of the two media, the optimum components for cell growth were predicted. Then, the growth medium of the DF1 cells was optimized rapidly and efficiently using the DOE statistical design method. The growth of DF-1 cells was improved, and the production of the IBDV vaccine was significantly enhanced, with the optimized medium. This study provided guidance for the production of viral vaccines using DF- 
1 cell, and a set of schemes for the rapid design and optimization of the cell growth medium based on metabolite analysis.

\section{Conflicts of interest}

There are no conflicts to declare.

\section{Acknowledgements}

This study was funded by the Chinese National Programs for High Technology Research and Development (2015AA020801).

\section{Notes and references}

1 J. Schaefer-Klein, I. Givol, E. V. Barsov, J. M. Whitcomb, M. VanBrocklin, D. N. Foster, M. J. Federspiel and S. H. Hughes, Virology, 1998, 248, 305-311.

2 A. M. Levy, O. Gilad, L. Xia, Y. Izumiya, J. Choi, A. Tsalenko, Z. Yakhini, R. Witter, L. Lee, C. J. Cardona and H. J. Kung, Proc. Natl. Acad. Sci. U. S. A., 2005, 102, 14831-14836.

3 C. W. Lee, K. Jung, S. J. Jadhao and D. L. Suarez, J. Virol. Methods, 2008, 153, 22-28.

4 K. A. Moresco, D. E. Stallknecht and D. E. Swayne, Avian Dis., 2010, 54, 622-626.

5 Y. Q. Wang, X. L. Qi, H. L. Gao, Y. L. Gao, H. Lin, X. Q. Song, L. Pei and X. M. Wang, J. Virol. Methods, 2009, 157, 205-210.

6 K. Rekha, C. Sivasubramanian, I. M. Chung and M. Thiruvengadam, BioMed Res. Int., 2014, 494835.

7 S. Dietmair, M. P. Hodson, L. E. Quek, N. E. Timmins, P. Chrysanthopoulos, S. S. Jacob, P. Gray and L. K. Nielsen, Biotechnol. Bioeng., 2012, 109, 1404-1414.

8 N. Guan, J. Li, H.-d. Shin, J. Wu, G. Du, Z. Shi, L. Liu and J. Chen, Metabolomics, 2014, 11, 1106-1116.

9 B. Wang, J. Liu, H. Liu, D. Huang and J. Wen, J. Ind. Microbiol. Biotechnol., 2015, 42, 949-963.

10 S. Klein and E. Heinzle, Wiley Interdiscip. Rev.: Syst. Biol. Med., 2012, 4, 261-272.

11 S. Dietmair, M. P. Hodson, L. E. Quek, N. E. Timmins, P. Gray and L. K. Nielsen, PLoS One, 2012, 7, e43394.

12 R. V. Kapoore, R. Coyle, C. A. Staton, N. J. Brown and S. Vaidyanathan, Metabolomics, 2015, 11, 1743-1755.

13 C. Korneli, C. J. Bolten, T. Godard, E. Franco-Lara and C. Wittmann, Biotechnol. Bioeng., 2012, 109, 1538-1550.

14 P. K. Chrysanthopoulos, C. T. Goudar and M. I. Klapa, Metab. Eng., 2010, 12, 212-222.

15 R. L. Plackett and J. P. J. B. Burman, Biometrika, 1946, 33, 305-325.
16 G. E. P. Box and D. W. J. T. Behnken, Technometrics, 1960, 2, 455-475.

17 Y. H. Zhang, H. M. Huang, S. S. Xu, B. Wang, J. H. Ju, H. R. Tan and W. L. Li, Microb. Cell Fact., 2015, 14, 11.

18 S. M. Paixao, P. D. Teixeira, T. P. Silva, A. V. Teixeira and L. Alves, New Biotechnol., 2013, 30, 598-606.

19 G. Ebrahimipour, H. Sadeghi and M. Zarinviarsagh, Molecules, 2017, 22, 15.

20 J. Si, G. Meng, Y. Wu, H. F. Ma, B. K. Cui and Y. C. Dai, Int. J. Biol. Macromol., 2019, 124, 1186-1196.

21 T. Y. Yun, R. J. Feng, D. B. Zhou, Y. Y. Pan, Y. F. Chen, F. Wang, L. Y. Yin, Y. D. Zhang and J. H. Xie, PLoS One, 2018, 13, 21.

22 A. A. Abd El Aty, H. R. Wehaidy and F. A. Mostafa, Carbohydr. Polym., 2014, 102, 261-268.

23 F. Cui and L. Zhao, Int. J. Mol. Sci., 2012, 13, 10630-10646. 24 K. A. Lawton, A. Berger, M. Mitchell, K. E. Milgram, A. M. Evans, L. Guo, R. W. Hanson, S. C. Kalhan, J. A. Ryals and M. V. Milburn, Pharmacogenomics, 2008, 9, 383-397.

25 M. M. Bradford, Anal. Biochem., 1976, 72, 248-254.

26 J. Chong, O. Soufan, C. Li, I. Caraus, S. Li, G. Bourque, D. S. Wishart and J. Xia, Nucleic Acids Res., 2018, 46, W486-W494.

27 J. Xia and D. S. Wishart, Curr. Protoc. Bioinf., 2016, 55, 14.10.1-14.10.91.

28 C. Li, S. Q. Sun, D. Park, H. O. Jeong, H. Y. Chung, X. X. Liu and H. M. Zhou, Int. J. Biol. Macromol., 2011, 49, 910-916.

29 C. L. Hsieh, H. E. Wang, W. J. Tsai, C. C. Peng and R. Y. Peng, Toxicology, 2012, 291, 32-42.

30 R. M. Hoffman, Biochim. Biophys. Acta, 1984, 738, 49-87.

31 A. M. Hosios, V. C. Hecht, L. V. Danai, M. O. Johnson, J. C. Rathmell, M. L. Steinhauser, S. R. Manalis and M. G. Vander Heiden, Dev. Cell, 2016, 36, 540-549.

32 A. Leon-Del-Rio, V. Valadez-Graham and R. A. Gravel, in Annual Review of Nutrition, ed. P. J. Stover and R. Balling, Annual Reviews, Palo Alto, 2017, vol. 37, pp. 207-223.

33 R. Banerjee and S. W. Ragsdale, Annu. Rev. Biochem., 2003, 72, 209-247.

34 K. Gruber, B. Puffer and B. Krautler, Chem. Soc. Rev., 2011, 40, 4346-4363.

35 W. M. Liu, F. X. Shi, L. Z. Lu, C. Zhang, Y. L. Liu, J. Zhang, Z. R. Tao, J. D. Shen, G. Q. Li, D. Q. Wang, J. J. Li and Y. Tian, Mol. Cell. Biochem., 2011, 352, 19-24.

36 Z. Rehman, S. Naz, R. U. Khan and M. Tahir, World's Poult. Sci. J., 2017, 73, 823-830. 